\title{
Mapping the emotional terrain of midwifery: What can we see and what lies ahead?
}

\author{
Billie Hunter \\ Institute for Health Research, \\ School of Health Sciences, \\ Swansea University, \\ Floor 2, Vivian Tower, SA2 8PP, UK \\ E-mail: b.j.hunter@swansea.ac.uk
}

\begin{abstract}
Greater understanding of midwives' emotion work could potentially improve practitioner/client and collegial relationships, and inform future organisation and delivery of maternity care. However, midwives' emotion work remains relatively unrecognised and under-investigated. Drawing on a previous paper (Hunter, 2001), I contrast previous and current knowledge in the field. Four key areas appear significant: impact of work context and organisation; midwife-woman relationships; collegial relationships; emotion work as coercion or gift? More research is needed into how midwives manage emotions and learn emotion management skills. Trends towards "high tech but low care solutions" (Kirkham, 2009, p.237) have implications for future emotion work study.
\end{abstract}

Keywords: emotion work; boundary work; midwife-woman relationships; collegial relationships; care deficit; emotion; midwifery; work organisation.

Reference to this paper should be made as follows: Hunter, B. (2010) 'Mapping the emotional terrain of midwifery: What can we see and what lies ahead?', Int. J. Work Organisation and Emotion, Vol. 3, No. 3, pp.253-269.

Biographical notes: Billie Hunter has been a midwife for 30 years, working in NHS, voluntary sector and independent midwifery settings, before moving into midwifery education and research in 1996. She has published widely and is the co-author of a popular oral history of midwifery: The Midwife's Tale (Leap and Hunter, 1993) and co-editor with Ruth Deery of Emotions in Midwifery and Reproduction (2009).

\section{Introduction}

Across cultures, childbirth is acknowledged to be an emotionally laden event (Davis-Floyd and Sargent, 1997). Any cursory trawl of the popular media reveals that the birth of a baby is the source of high emotion, a time when "even grown men cry". Many books, aimed at both parents and midwives, provide a wealth of anecdotal evidence illustrating the range of emotions that may be experienced - from joy and delight to extremes of fear and anxiety (Gaskin, 2002; Kitzinger, 2008). This emotional life event could also be expected to affect the main caregivers, that is, the midwives for whom it is the focus of their day-to-day work. In this paper I provide a critical review of 
the literature relating to the emotional aspects of midwifery work, drawing on evidence from a range of national and international research studies. My aim is to provide a "state-of-the-art" map of this field as it currently stands, identifying key issues and debates, whilst also noting the areas that remain unexplored and largely invisible. These remain as a challenge for future study.

Midwifery is an interesting example of a gendered occupation, in which a predominantly female workforce provides sensitive and intimate care to a predominantly female client group. ${ }^{1}$ There is a strong ideological commitment to a social model of health, which views birth as a normal life event of social and cultural significance (Davis-Floyd and Sargent, 1997; Downe and McCourt, 2004). The relational aspects of care are given prominence. Midwifery is characterised by its ideological emphasis on 'woman-centred care' and autonomous practice, which co-exist uncomfortably within contemporary healthcare systems which favour 'efficient' processing of clients and standardisation of care (Hunter, 2004).

The emotion work of midwives is a comparatively new area for research; in comparison with nursing, it has (rather surprisingly) gone largely unrecognised and under-investigated. Given the many differences between two occupations, there are likely to be different sources of emotion work. In this paper, I argue that greater understanding of midwives' emotion work has practical as well as theoretical implications. In particular, it has the potential to improve relationships between clients and practitioners, and between colleagues. It can also provide insights to inform the future organisation and delivery of maternity care.

As a starting point, I draw on a previous paper (Hunter, 2001) in order to contrast our understanding 'then and now'. The first part of the paper summarises the knowledge base in 2001. In the second part, current thinking in the field is considered and key themes from contemporary studies are identified. Finally, current gaps in knowledge are noted. I ask how we can make this 'hidden terrain' more visible and better understood, and whether these insights into the emotion work of midwives have wider implications.

\section{Previous knowledge base}

In 2001, I published a critical review of existing knowledge relating to the emotion work of midwives (Hunter, 2001), based on a literature search undertaken during 1998-1999 using Medline, CINAHL, BIDS and MIDIRS databases. The paper drew attention to the remarkable lack of research in this area, given the emotional nature of childbirth. The few emotions-related studies which did exist focused on stress and burnout (Sandall, 1997; Mackin and Sinclair, 1998), thus providing insights into only some aspects of emotion in the workplace. However, research studies dealing with other elements of midwifery work did provide some interesting and unexpected insights: for example, a study of the midwife's role in relation to pain relief during labour suggested that witnessing childbirth pain could be distressing for attending midwives (Niven, 1994).

This lack of research investigation prompted my own doctoral study, which used an ethnographic approach to explore the sources of emotion work in midwifery and how midwives manage their emotions in the workplace (Hunter, 2002). The critical review (Hunter, 2001) was written prior to commencing fieldwork. It was used to set out tentative suggestions for possible sources of emotion work in midwifery, based on the 
available literature and informal discussions with midwives. I argued that contemporary midwifery presented a number of features especially likely to generate emotion, which related both to the way that maternity care was organised and delivered, and to the very 'essence' of midwifery work.

The organisation and delivery of UK maternity care was undergoing significant change at the time of the study. Midwife-led schemes providing woman-centred care were being developed in an ad hoc manner, in response to the recommendations of the Changing Childbirth Report (Department of Health (DoH), 1993). The schemes aimed to increase choice and control for women, and to provide continuity of care, either from a known midwife or team of midwives. I postulated that increased continuity of care had the potential to increase midwives' emotion work: firstly, because 'on call' systems could blur work-home life boundaries; secondly, because women's expectations of the midwife could be raised unrealistically; and finally, because it was probable that this idealised way of working would be difficult to achieve in practice - that is, there would be dissonance between ideals and reality.

The Changing Childbirth Report (Department of Health (DoH), 1993) also recommended that childbirth be treated as a normal event wherever possible, and that the role of midwives as experts in normal childbirth should be reclaimed. This re-positioning had implications for the occupational roles of midwives and doctors and, consequently, for the division of labour. Historically, the occupational territories of midwives and doctors have been keenly contested, leading to role ambiguity and 'turf wars' (Donnison, 1977; Kitzinger et al., 1990). I argued that reconfiguration of occupational territories could exacerbate these tensions and increase the need for emotion work.

Apart from these changes in the organisation and delivery of care, the review also noted a number of characteristics related to the 'essence' of the midwife's work which were likely to require emotion management. In particular, childbirth pain, bodywork and sexuality were identified as likely sources of emotion work, requiring midwives to manage their own feelings, as well as the emotions of the woman and her partner.

Midwives attend women as they engage in one of life's most challenging and intimate events, where high levels of emotional display are the norm. For example, most labouring women experience intense sensations and pain as part of the normal physiological process. Studies suggest that nurses caring for ill and distressed patients use withdrawal and distancing as coping strategies (James, 1992; Smith, 1992). I wondered whether midwives caring for well women experiencing the physiological pain of childbirth, rather than the pathological pain of disease, draw on similar techniques. Research based evidence was lacking to answer my questions.

Midwifery also involves intimate 'bodywork' such as examinations of the abdomen, breasts and vagina, and dealing with breast milk, blood, urine and faeces (Twigg, 2006). Again, we have some insights into how nurses negotiate intimate care, which suggests that they remind themselves of the illness of their patients in order to manage their personal reactions (Lawler, 1991). Midwives do not have this 'fall back', and thus midwifery bodywork may present a different range of challenges, which will necessarily involve emotion work.

In addition midwifery, by its very nature, entails engagement with human sexuality. Childbirth is in itself evidence of sexual behaviour and for some women it is experienced sexually. The recent emphasis on birth as a natural life event, rather than a medical condition to be managed and sanitised, has further opened up opportunities for its sexual dimensions to be discussed. I speculated that this could prove challenging for many 
midwives, for whom the topic is taboo. These issues are not widely acknowledged, and I observed that discussions in the literature of midwives' personal reactions to pain, bodywork and sexuality were notable by their absence.

\section{Present: What do we know now?}

In the second section of the paper, I bring the discussion up to date by reviewing recent research related to emotion in midwifery work, noting the growing number of UK and international studies over the past decade.

The review was undertaken by searching the national and international literature using Medline, CINAHL, and MIDIRS databases for the period 1999-2008, using the keywords midwifery, maternity care, emotion work, emotional labour, relationships. Reference lists were examined to identify further relevant articles and texts. In addition, I was in the privileged position of co-editing a collection of related studies ("Emotions in Midwifery and Reproduction", Hunter and Deery, 2009) which facilitated access to very recent studies which had not previously been published.

It was notable that most of the reviewed studies employed qualitative methodologies, in particular ethnographic approaches. This is entirely appropriate, given that they intended to explore the experiences of midwives and/or their clients, and to provide rich, in-depth accounts of these experiences within their cultural contexts. Ethnography allows us to observe what people do, as well as what they say they do, and can bring many unexpected insights. Its appropriateness as a research method is suggested by its use in many of the classic studies of emotional labour (Hochschild, 1983; Smith, 1992; James, 1992).

It was interesting to note that only some studies started out with the explicit intention of investigating emotion; others found this evidence unexpectedly. For example, whilst my own research had the specific aim of exploring the sources of midwives' emotion work and how these emotions were managed (Hunter, 2002), Deery (2005) set out to investigate the support needs of community midwives and the implementation of a clinical supervision system. A key unexpected finding in her study was the extensive emotion work which midwives described, both in their interactions with colleagues and with clients, and the range of professional 'performances' which they employed.

Four main themes were identified from this review:

- the impact of context and organisation of maternity care

- emotion work in midwife-woman relationships

- emotion work in collegial relationships

- emotion work as coercion or gift?

These themes are discussed in turn and contrasted with those issues identified in the earlier paper (Hunter, 2001), using extracts from research data to illustrate the discussion. 


\section{The impact of context and organisation of maternity care}

It was notable that many of the studies which unexpectedly 'discovered' midwives' emotion work were those whose primary focus was service delivery and organisation (i.e., Stevens and McCourt, 2002; Dykes, 2005). The scale of the workplace and the model of care provision appear to be critical factors for emotion management, affecting how emotions are experienced and regarded. In particular, a key element is whether there is the potential for establishing meaningful, supportive relationships with clients and colleagues.

The dominant approach in westernised maternity care is for most services to be centralised into large hospital units. Critics argue that this industrial model of childbirth is damaging for both receivers and providers of care (Hunter et al., 2008; Kirkham, 2009). The prevailing 'production-line' approach requires conformity, task orientation and suppression of emotions in order to ensure that institutional goals are reached. Care becomes reductive and fragmented, and the work of the midwife becomes goal orientated rather than client focused. This system clearly militates against relationship formation.

Emotion work is needed to manage the challenges of providing an adequate level of care against the odds, and to deal with the dissonance experienced when the reality of the workplace contrasts so vividly with occupational and professional ideals (Lipsky, 1980; Hunter, 2004), as in this account:
"I went through a stressful time, because I had to make an adjustment personally that I could not expect to do the same things that I did in the past (...) I had to become more technical than I had ever been, and I had ... to do things faster. That bothered me a lot. I know I went through a real depression almost, and then finally I had to let go of it. I couldn't do things as thoroughly as I liked. I changed my attitudes and my expectation. That was very difficult." (Bone, 2009, p.68)

Current workforce shortages and a rising birth rate in the UK (Davis, 2008) exacerbate these difficulties. As Hochschild (1983, p125) explains, in situations where the "conveyer belt" speeds up, "the job of 'enjoying the job' becomes harder and harder" and workers reduce their emotion work and become detached (Hochschild, 1983). This withdrawal of emotional labour is aptly described as "going into robot" (Hochschild, 1983, p.129), and resonates with the account above from Bone's study. The literature provides many descriptions of midwives' 'robotic' and 'absently present' behaviour, both from the perspectives of midwives and women receiving care (Berg et al., 1996; Deery, 2009; Edwards, 2009).

In her critical ethnography of postnatal care, Dykes $(2005,2009)$ found that pressures of time, staff shortages and rapid client turnover "at the end of the production line" created emotion work for midwives. The emphasis was on efficient processing and rationing of care rather than engaging in meaningful relationships. This created frustrations and dissatisfaction for both mothers and midwives:

Midwife: “There isn't the time needed to help women, let alone give them
appropriate breastfeeding support ... you can't do that when you're busy.
You might have several antenatals, an early labourer, post sections and we're
even the overspill for gynae ... you just can't do it." (Dykes, 2009, p.93)

Mother: "They (midwives) seem to be pressurised, panicky and anxious." (Dykes, 2009, p.94) 
Time is a major factor in maternity care. Time is needed for midwives to establish rapport and build up relationships with women and their partners; to assess what is needed and to offer that in an unhurried manner. It is also needed for the physiological process of childbirth to unfold. However, this 'process time' is counterculture to the 'clock time' of the hospital conveyor belt, which requires activities to be 'productive' and resource efficient (Davies, 1994).

Bone's (2009) research takes these concerns about the consequences of conveyor belt care one stage further. Observing the work of maternity nurses in the US managed care system, she noted how the emotional and physical support needs of labouring women presented a challenge to the desired 'streamlined' approach. It was evident that epidural analgesia was used as a solution to this problem, as it reduced the need for one to one care and thus decreased the demands on an over-stretched workforce. A labour ward of women who are all attached to epidurals and electronic monitors can be 'managed' by technicians, often operating at a distance from a central surveillance station. As a result, the emotion work skills of childbirth attendants become devalued and eroded, and the focus of work becomes task related rather than relational. This is clearly a desolate situation for both women and their carers.

Bone draws on what Hochschild (2003) calls the contemporary 'care deficit' to analyse this situation. Hochschild proposes that in many industrialised countries, the need for care has expanded at the same time as the supply has contracted. It is also the case that public expectation of receiving high quality, sensitive care has increased, and this is particularly the case in maternity care. However, in market driven economies, health care has become viewed as a commodity, where cost effectiveness is crucial. Paying for care from highly skilled workers is expensive and all too often resources are reduced in the name of efficiency. Such cost cutting may take the form of reducing the number of skilled workers, employing cheaper, less highly trained workers or, as Bone suggests using the 'postmodern' solution of learning to live without much care. From this perspective "the issue of epidural analgesia (...) could be seen as a powerful, highly embodied metaphor for the post-modern solution to the care deficit" (Bone, 2009, p.57).

However, there are positive signs. A growing body of evidence suggests that "small is beautiful" when it comes to providing high quality maternity care, whether this refers to the smallness of the unit (Walsh, 2006) or smallness of work scale e.g., caseload midwifery (McCourt and Stevens, 2009). Such schemes are popular with mothers and midwives. Smallness of scale facilitates the formation of meaningful relationships, both between midwives and clients, and between midwives and colleagues. Relationships which are mutual and reciprocal provide emotional support, and facilitate the development of trust, an essential ingredient for effective team working and safe practice (Walsh, 2006; Hunter et al., 2008).

As the co-existence of these differing approaches to delivery of care suggests, they are underpinned by contradictory models of maternity care, in particular, a social model and a bio-medical model. In UK maternity care for example, there is a growing tension between the normalising approach to childbirth promoted by 'new' midwifery on the one hand and the risk averse culture of the NHS on the other (Walsh et al., 2004). Whilst UK midwives are urged to work in ways which promote normal birth and support individual women in achieving this, health service managers have a disparate aim of reducing risk via protocols and policies aimed at standardisation of care. These 'conflicting ideologies' (Hunter, 2004) are likely to create tensions and ambiguities for midwives, which require management of emotions. 


\section{Emotion work in midwife-woman relationships}

There is a strong body of international evidence which demonstrates the significance of the midwife-mother relationship both to women (Kennedy, 1995; Berg et al., 1996; Halldorsdottir and Karlsdottir, 1996; Kirkham, 2000) and midwives (Sandall, 1997; Walsh, 2007; Olafsdottir, 2009).

A range of emotions may be experienced within this relationship, for example: joy, calm, affection, humour, frustration, fear, sadness, anger; and emotion work may be needed on both the part of midwife and woman (Edwards, 2009). Relationships can be mutually satisfying, enriching and meaningful; or alternatively, frustrating, impoverished and distressing. The latter situation, however, has received limited research attention. Professional ideologies which emphasise the woman-centred nature of midwifery work have tended to romanticise the relationship and make it difficult to explore this shadow side (Hunter, 2006).

The evidence suggests that when meaningful relationships can be created, they are highly valued by both practitioners and clients. A key factor is the presence of reciprocity: that is, mutual 'give and take'. When this exists, both midwives and mothers feel recognised and valued as individuals (Hunter, 2006; McCourt and Stevens, 2009). In the words of a midwife, in such situations "from an emotional point of view you give more but you get more" (Hunter, 2006, p.317).

Analysis of women's accounts of their positive encounters with midwives reveals the issues which are of primary importance. In many of these narratives, there is clear evidence of the emotion work in which the midwife is engaged. For example:

"It's the midwife's job to keep everything safe. She's the anchor that helps you go off into that altered state (...) they anchored me and allowed me to feel safe through all that." (Anderson, 2000, p.101)

“... she [midwife] stayed as cool as a cucumber, which you know, if she hadn't, if she's at any point suggested that I wasn't going to make it then that would have had a huge influence on me, you know, "cos I would have said “oh, great you agree. Okay, I can’t do it.” (Edwards, 2009, p.38)

But experiences are not always positive, as the following accounts illustrate:

\footnotetext{
"But I felt as she (midwife) always came just two minutes too late ... I felt as if half of her was still in the other room." (Berg et al., 1996, p.13)

"She didn't spend much time with me, coming in and out ... she seemed quite distracted, like her mind wasn't really in the room." (Anderson, 2000, p.102)

"I felt a little bit invisible. It was a bit smiley and a bit formulaic." (Edwards, 2005)
}

In the narratives of these women, positive experiences occurred when emotions were managed effectively (both their own emotions and those of the midwife). There is a sense of meaningful connection, trust and authenticity, evident in the language used. This leads to a feeling of 'emotional safety', which is arguably a key component of clinical safety. Maintaining this authentic connection throughout the duration of a long labour requires emotion work on the part of the midwife. In my own research (Hunter, 2002), I observed how midwives reported feeling emotionally exhausted after supporting women in labour. Even in a normal physiological labour, 'being there' for women through the physical and emotional upheavals of childbirth (Berg et al., 1996) requires intensive emotion work 
on behalf of the midwife. If labour becomes problematic, this emotion work is inevitably increased (Hunter, 2009; Olafsdottir, 2009).

In contrast, negative encounters are experienced when midwives appear to be distracted, disinterested and disengaged. This may be experienced by women as unfriendly and even hostile behaviour (Redshaw et al., 2007) or more commonly, a feeling that the midwife is 'absently present' (Berg et al., 1996) with her responses lacking authenticity, as in the accounts above. In such situations, there is apparently little effective emotion work being undertaken. It is likely that many of these negative encounters are the result of conveyor belt care and the resulting care deficit.

It is evident from the literature that it is not only women who value the midwife-woman relationship. Meaningful relationships with clients are also essential for midwives' job satisfaction (Sandall, 1997; Kirkham et al., 2006). They are a key ingredient in what midwives love about their work and contribute to the midwife's sense of self, the feeling they are "a person not a role" (McCourt and Stevens, 2009, p.19). Again, the level of reciprocity is crucial. When the environment of care is conducive to reciprocity, even if emotion work is needed, it is experienced positively by the midwife, who feels that she is able to 'make a difference'. It is where this reciprocity is missing that emotion work is experienced negatively (Hunter, 2006).

\section{Emotion work in collegial relationships}

Relationships between midwifery colleagues are also a major source of emotion work (Hunter, 2005; Deery and Kirkham, 2007). This is particularly the case for midwives working within institutional settings, for whom colleagues form the "primary reference group" (Lipsky, 1980, p.47), as opposed to those in community-based practice, for whom relationships with clients are of more significance (Hunter, 2005).

Whilst positive collegial relationships have been described, with midwives offering each other mutual support (both physical and emotional) within workplace cultures typified by trust and reciprocity, these are relatively rare descriptions. Walsh (2006, 2007) provides fascinating examples of the social capital built up within a freestanding birth centre. He identified a number of features which contributed to a highly supportive workplace: staffing of the unit was flexible, home life and work boundaries were relatively blurred and the midwives met regularly out of work for social activities, contributing to the sense of collective identity. As a result, when midwives were experiencing personal crises, the level of collegial support was high. Many of the staff alluded to working at the birth centre as being akin to being in a family and a strong sense of shared communitarian values runs throughout Walsh's fieldwork observations (Walsh, 2006).

Such descriptions, however, are far from typical. Negative experiences abound, with many studies revealing that lack of workplace support is commonplace within UK midwifery (Kirkham, 1999; Ball et al., 2002). This lack of support manifests in many ways. For some midwives, the key concern is not being valued as an individual but merely as a source of physical labour, moved about according to the demands of the workplace. As in the following account, in such situations midwives feel as though they are 'just a thing': 
"You're completely invisible, you run in, take the baby, come out (...) you're just a thing that came in, did them up a bit and then disappeared again." (Hunter, 2002: Focus Group with community-based team midwives)

For others, the concerns are more extreme and distressing. There is evidence of a bullying culture in many maternity units (Ball et al., 2002), which leads to low morale and dissatisfaction. Studies of UK midwifery have shown that lack of support is a key factor cited by midwives who decide to leave the profession. Conversely, good quality support is a reason for staying (Ball et al., 2002; Kirkham et al., 2006).

Clearly, negative relationships with colleagues will require emotion work. In my own study, I found that such situations left midwives feeling anxious and demoralised. This was particularly the case for less experienced midwives, as in the following account:

\footnotetext{
"The most difficult thing for me is people (...) often colleagues - colleagues can be the biggest thing, this animosity from senior staff, this bullying (group agreement) - the issue of bullying at work (...) and it can crush, it can crush an atmosphere, it can crush a situation, it can crush a relationship with a woman, it can crush you professionally." (Voices in agreement) (Hunter, 2002: Focus Group with hospital midwives)
}

Why this lack of collegial support should be so prevalent in midwifery is puzzling. Some insights are offered by Kirkham (1999) and Kirkham and Stapleton (2000), whose research into the culture of midwifery in the UK National Health Service (NHS) throws light on the prevailing social norms. Kirkham (1999, p.736) characterises midwifery as a hierarchical, 'blame culture' in which midwives are reluctant to challenge or confront, but rather "do good by stealth". There is a lack of awareness of the commonality of negative experiences, which further isolates individual midwives and limits opportunities for collective action. Kirkham argues that this culture needs to be understood as genderised, underpinned historically by an ethic of service, self-sacrifice and conformity. Such situations are fertile breeding grounds for 'horizontal violence' (Leap, 1997), whereby oppressed groups internalise the views of those in power and direct criticism inwards, in order to suppress the views of group members that are considered to be deviant.

Many studies indicate that midwives are reluctant to seek support from colleagues for fear of rejection or ridicule. Impression management (Goffman, 1969) is employed to ensure that the professional mask is maintained and credibility with co-workers is preserved (Deery, 2009). The emotional norm in many maternity units appears to be that of 'affective neutrality' (Parsons, 1951): emotions are suppressed and disguised to ensure that the workplace remains as emotionally neutral as possible and the smooth running of the maternity unit is not impeded. This takes its toll on maternity care staff (Deery and Kirkham, 2007; Bone, 2009). In other settings such as birth centres and community midwifery, however, 'affective awareness' is valued; that is, the expression of emotion and sharing of feelings (Hunter, 2006; Walsh, 2007). Thus, how emotions are managed - or not managed - appears to be context related (Hunter, 2009). Collegial disharmony and conflict are more often reported within large-scale institutional settings than in smaller birth centres or community-based maternity care.

The current shortage of midwives in the UK also jeopardises the creation of supportive collegial relationships (Davis, 2008). Midwives describe the disruption which ensues when they are re-located to busy areas at a moment's notice in order to fill gaps in service provision (Hunter, 2004; Dykes, 2009). As a result, teams are often transitory 
groupings formed for the duration of only one shift and consisting of unfamiliar workmates. This sense of impermanence and unfamiliarity is exacerbated by the growing use of temporary agency and 'bank' staff. Relationships are fragmented and there is a consequent reduction in mutual trust and social capital. It is not difficult to imagine that the camaraderie and reciprocity described by Walsh $(2006,2007)$ will be an unlikely occurrence in such situations.

Not surprisingly, impoverished collegial relationships take their toll on students and newly qualified staff. In my own study, novice midwives gave vivid accounts of working within a hierarchical and unsupportive culture, and the demoralisation and de-motivation this caused (Hunter, 2005). It appeared that collegial conflicts were often underpinned by differing ideologies. The more junior midwives advocated a non-interventionist, 'with woman' approach focusing on the needs of individual women. In the views of more senior midwives, this was idealistic and unachievable. Their focus was 'with institution', aimed at task completion and meeting the needs of the institution. These senior midwives controlled the workplace via a system of unwritten rules, idiosyncratic practices, criticism and sanctions, which left the inexperienced midwives bewildered and wrong-footed at best, and demoralised and distressed at worst. The resulting collegial disharmony required extensive emotion work on the part of the junior midwives, as the following account suggests:

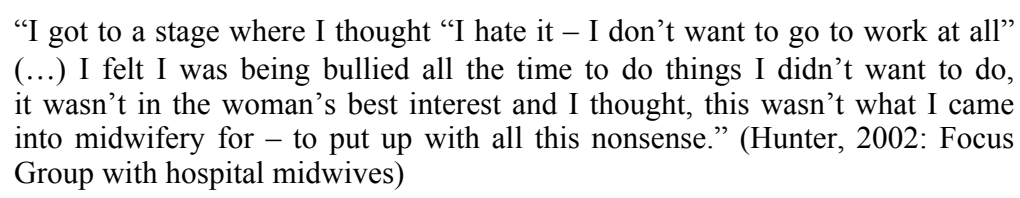

"I got to a stage where I thought "I hate it - I don't want to go to work at all" (...) I felt I was being bullied all the time to do things I didn't want to do, it wasn't in the woman's best interest and I thought, this wasn't what I came into midwifery for - to put up with all this nonsense." (Hunter, 2002: Focus Group with hospital midwives)

The recent literature on collegial relationships in maternity care focuses mainly on relationships between midwives. There is less contemporary evidence relating to relationships between midwives and doctors. As noted earlier, tensions between midwives and doctors have historically been underpinned by contested territories and struggles for occupational jurisdiction. But, whilst these relationships were the topic of earlier papers and texts (Donnison, 1977; Kitzinger et al., 1990), recent evidence is limited. This should not be taken as an indication that these tensions have receded, however, as inter-professional tensions and ineffective team working have been noted in recent reports (Healthcare Commission, 2008; Redshaw et al., 2007). There is scope for additional research in this area.

\section{Emotion work as gift or coercion?}

Many of the studies reviewed reveal negative aspects of emotion work, noting how this is often related to healthcare systems which privilege efficiency, effective processing and task completion. To achieve these aims, client compliance is needed, and as a result, emotion work becomes coercive (Kirkham, 2009). For example, interviewing women about their experiences of home birth, Edwards observes how midwives can

\footnotetext{
"evoke feelings of fear, uncertainty, selfishness, guilt, blame, irrationality and even silliness, or threaten withdrawal, to make sure women behave appropriately - that is, in line with local polices and practices." (Edwards, 2009, p.39)
} 
Many authors observe the tragedy of this situation, as it moves both mother and midwife "away from the deeper emotional connections forged by the experience of natural childbirth" (Bone, 2009, p.69) and leads to "social and emotional impoverishment" (Kirkham, 2009, p.237).

It is important, however, not to paint too dismal a picture. There are also studies which show that emotion work can be pleasurable, usually in situations where levels of autonomy are high and where relationships are valued (Walsh, 2006, 2007; McCourt and Stevens, 2009). Olafsdottir (2009) for example, draws on her narrative analysis of the stories of Icelandic midwives to demonstrate how midwives value the "strong emotions which arise from close connections with the woman and her family" (Olafsdottir, 2009, p.195). She argues that these emotional experiences are necessary for midwives to learn the skills of expert practice, as they form part of midwives' 'inner knowing' about their work. There are links here with the work of Walsh (2006, 2007), who provides many examples of midwives managing emotions in ways that enhanced the richness and quality of the childbirth experience. In both studies, midwives were able to exercise autonomy not only in their clinical practice, but also in their emotion work. In such situations, emotion work, whilst demanding, is also rewarding. As Bolton (2000) argues, it can become a 'gift' rather than a chore. Midwives feel that they have 'made a difference' to the emotional wellbeing of the woman and her family, and often that they have contributed to the greater social capital.

\section{Summary of current knowledge}

In summary, the current knowledge base suggests that there are several key areas of significance when considering the emotion work of midwives: the impact of context and organisation of maternity care; midwife-woman relationships and collegial relationships. None of these themes exist in isolation - they are all interwoven so that one inevitably impacts on the others. For example, the context in which care is provided has implications for the quality of relationships between midwives and women, and also for relationships between midwives. It may also affect whether the emotion work that is performed is experienced as coercive or as a gift.

To return to the possible areas identified in the earlier paper (Hunter, 2001): it would seem that some of my tentative suggestions now have a substantive evidence base to support them, whilst for others the evidence is less clear.

\section{To consider them in turn:}

My original suggestion that continuity of care could lead to increased emotion work for midwives does not appear to be supported by the evidence. Although some studies have shown that continuity of care schemes may create stresses for midwives because of blurred work/life boundaries and unsustainable workloads (Sandall, 1997; Green et al., 1998), other findings dispute this. McCourt and Stevens (2009), for example, show that one to one caseload schemes increase midwives' autonomy and are thus a source of job satisfaction and emotional reward, valued by midwives and women. It is suggested that the emotional demands of providing continuity of care are balanced by the emotional rewards of "knowing and being known" (McCourt and Stevens, 2009, p.18), and that the issue is much more complex than may be surmised at first glance. There is also evidence that the problems which have been reported frequently stem not from the introduction of 
continuity of care schemes per se, but from the type of scheme that has been created or the manner in which it has been introduced (Green et al., 1998; McCourt and Stevens, 2009). Team midwifery schemes, for example, when large groups of midwives provide care for large caseloads of women, do not offer the same opportunities for relationship formation and autonomous practice, and may, in fact, increase occupational stress (Sandall, 1997).

I also postulated that relationships between midwives and doctors could be a key source of emotion work, as a result of role ambiguity and longstanding 'turf wars'. Whilst there is evidence that this continues (Healthcare Commission, 2008), what is much more notable is the strong (and unexpected) evidence demonstrating tensions between midwives, experienced as horizontal violence and responsible for low morale and attrition.

In relation to emotion work arising from the 'essence' of midwifery work, the evidence base remains relatively scanty. There is little evidence that the high levels of displayed emotion which are common during childbirth are problematic for midwives, or that they require substantial emotion work on their part. However, it may be that the right research questions have yet to be asked, or perhaps that these data have yet to be accessed via appropriate research designs. My own observational fieldwork of midwives suggests that midwives do experience their work as emotional, but that this is rarely articulated explicitly in interview data.

Similarly, little is known about midwives' reactions to intimacy, 'body work' and sexuality. My own study provided some evidence that student midwives could find providing intimate care for another woman's body emotionally challenging. However, as only a small number of participants raised this issue, it was not possible to draw any conclusions. A recent study of midwives' and women's experiences of vaginal examinations provides some further insights (Stewart, 2005), and suggests that intimacy and body work are issues worthy of more study.

I also suggested that caring for women experiencing childbirth pain could be distressing for midwives. Again, our knowledge base is very limited. There are interesting studies which have begun to explore these issues, for example, Bone's study of the use of epidurals in US maternity care and the important contemporary research of Nicky Leap (Leap and Anderson, 2004).

Thus, some of these potential sources of midwives' emotion work have been identified in recent research, whilst others have not. The question remains: is this because they are indeed not very significant, or is it because they are not as immediately obvious - or as open to being researched? Is it the case that further research would help us to uncover more about these issues, or are there other areas as yet unidentified needing exploration?

\section{Concluding thoughts}

In this paper I have provided a critical review of the literature relating to emotion work in midwifery, in order to map the 'emotional terrain' of midwifery. Using an earlier review as a baseline, I have drawn on a range of UK and international studies to set out the current picture and draw some tentative conclusions about the key issues. However, it is clear that there are many gaps in the knowledge base, and there is much more that we need to find out. 
We appear to have some insights into sources of emotion work for midwives, although there are still gaps and it is inevitable that these issues will change over time and between social and cultural contexts. Future developments in reproductive health, for example, such as new techniques for assisted conception and advanced foetal technologies have the potential to add to midwives' emotion work.

We know much less about how midwives manage emotions, both their own and those of the women and families they care for. Exploring these complex issues may require the use of imaginative research approaches, for example, the use of creative visual arts methods (Rose, 2001) in order to best explore this 'hidden terrain'. It is important, given the potential for midwives' emotion work to impact significantly on relationships with clients and colleagues, to find out more about 'expert' emotion workers. How do they achieve this expertise? Can it be learnt? Can it be taught? And if so, how? Given that midwifery students are taught in both clinical settings and within universities, which context is most appropriate for developing these skills? There is evidence that qualified midwives can provide students with supportive mentorship and act as inspiring role models (Finnerty et al., 2006). However, the demands of this role are rarely acknowledged; the skills of mentorship remain undocumented and hence largely invisible. As Pam Smith and I noted in a recent editorial in the International Journal of Nursing Studies, although there is more acknowledgement of emotion in midwifery and nursing curricula, there is a danger that this may be tokenistic if it is not reflective of authentic recognition at a grass roots level (Hunter and Smith, 2007).

These are important issues, as quite apart from their theoretical interest, they have significant implications for the quality of maternity care and wellbeing of midwives. How midwives experience and manage emotion will affect the care they give and hence the experiences of women, families and the wider community.

There may also be wider implications. Although there is much about midwifery that is special, in that it involves a predominantly female workforce giving intimate and highly skilled care to other women, there are broader insights of relevance to other workers. For example, gynaecology and fertility nurses may share some of the emotional challenges and rewards that result from the sensitivity and intimacy of the work. The tensions experienced because of 'conflicting ideologies' may also be experienced in other professional groups, where strongly held ideals of client centred approaches conflict with the reality of the job - for example, teaching, social work, medicine.

It is also the case that midwives are not alone in their experience of working in transitory teams, where trust and support cannot be assumed. This has implications for safe practice. Midwives' experiences may throw light on other occupations where temporary teams are the norm and safe practice is of paramount importance. Emotional safety may form a more significant component of clinical safety than we have previously acknowledged - it is highly probable that a workplace where colleagues trust and respect each other, will also be a safer one for all concerned. These are important issues to ponder, given the continued rise in childbirth interventions in much of the western world.

Midwives are also in a double bind - whilst they may want to privilege the emotion work aspects of their care, and move towards what Hochschild (2003, p.214) calls 'a warm modern' approach to the care deficit, organisational trends are moving in the opposite direction. The review suggests that emotion work continues to remains unrecognised and undervalued (John and Parsons, 2006; Hunter and Deery, 2009), particularly by those responsible for managing and commissioning healthcare. 
Streamlined care that responds to organisational imperatives, rather than to the emotional needs of clients and workers, leads to emotionally impoverished experiences for all concerned.

Recent changes in the pay structure for UK midwives illustrate this undervaluing, as higher status (and remuneration) is given to technical skills. Supportive care during labour is now often provided by maternity care assistants (who are much cheaper to train, and to employ than midwives) or by family members, leaving the midwives to dash from labour room to labour room, completing technical tasks. Women are encouraged to stay at home until they are well established in labour, and then to have epidurals when they arrive. It seems that Hochschild's postmodern solution - learning to do without - has reached the UK too.

Ironically, these "high tech but low care solutions" (Kirkham, 2009, p.237), whilst having short term appeal for budget holders, may prove to be more expensive in the long term. The evidence to date suggests that these solutions fail to meet the emotional needs of mothers and midwives, and may compromise patient safety. Future studies will need to explore both the physical and the emotional consequences of the care deficit within maternity care.

\section{References}

Anderson, T. (2000) 'Feeling safe enough to let go: the relationship between a woman and her midwife during the second stage of labour', in Kirkham, M. (Ed.): The Midwife-Mother Relationship, Macmillan Press, Basingstoke, pp.92-119.

Ball, L., Curtis, P. and Kirkham, M. (2002) Why Do Midwives Leave?, Women's Informed Childbearing and Health Research Group, University of Sheffield and Royal College of Midwives, London.

Berg, M., Lundgren, I., Hermansson, E. and Wahlberg, V. (1996) 'Women's experience of the encounter with the midwife during childbirth', Midwifery, Vol. 12, pp.11-15.

Bolton, S.C. (2000) 'Who cares? Offering emotion work as a 'gift' in the nursing labour process', Journal of Advanced Nursing, Vol. 32, No. 3, pp.580-586.

Bone, D. (2009) 'Epidurals not emotions: The care deficit in US maternity care', in Hunter, B. and Deery, R. (Eds.): Emotions in Midwifery and Reproduction, Palgrave Macmillan, Basingstoke, pp 56-72.

Davies, K. (1994) 'The tensions between process time and clock time in care-work: the example of day nurseries', Time and Society, Vol. 3, No. 3, pp.277-303.

Davis, K. (2008) 'Our maternity services are now at breaking point', The Guardian, 31 January, accessed online 25/2/2009: http://www.guardian.co.uk/commentisfree/2008/jan/31/comment. nhs

Davis-Floyd, R.E. and Sargent, C.F. (1997) Childbirth and Authoritative Knowledge: Cross-Cultural Perspectives, University of California Press, Berkeley, California.

Deery, R. (2005) 'An action research study exploring midwives' support needs and the effect of group clinical supervision', Midwifery, Vol. 21, No. 2, pp.161-176.

Deery, R. (2009) 'Community midwifery 'performances' and the presentation of self', in Hunter, B. and Deery, R. (Eds.): Emotions in Midwifery and Reproduction, Palgrave Macmillan, Basingstoke, pp.73-89.

Deery, R. and Kirkham, M. (2007) 'Drained and dumped on: the generation and accumulation of emotional toxic waste in community midwifery', in Kirkham, M. (Ed.): Exploring the Dirty Side of Women's Health, Routledge, London, pp.72-83. 
Department of Health (DoH) (1993) Changing Childbirth: Part 1: Report of the Expert Maternity Group, HMSO, London.

Donnison, J. (1977) Midwives and Medical Men, Schocken Books, London.

Downe, S. and McCourt, C. (2004) 'From being to becoming: reconstructing childbirth knowledges', in Downe, S. (Ed.): Normal Childbirth: Evidence and Debate, Churchill Livingstone, Edinburgh, pp.3-24.

Dykes F. (2005) 'A critical ethnographic study of encounters between midwives and breastfeeding women on postnatal wards', Midwifery, Vol. 21, pp.241-252.

Dykes, F. (2009) ' No time to care': Midwifery work on postnatal wards in England', in Hunter, B. and Deery, R. (Eds.): Emotions in Midwifery and Reproduction, Palgrave Macmillan, Basingstoke, pp.90-104.

Edwards, N. (2005) Birthing Autonomy: Women's Experience of Planning Home Births, Routledge, London.

Edwards, N.P. (2009) 'Women's emotion work in the context of current maternity services', in Hunter, B. and Deery, R. (Eds.): Emotions in Midwifery and Reproduction, Palgrave Macmillan, Basingstoke, pp.36-55.

Finnerty, G., Graham, L., Magnusson, C. and Pope, R. (2006) 'Empowering midwife mentors with adequate training and support', British Journal of Midwifery, Vol. 14, No. 4, pp.187-190.

Gaskin I.M. (2002) Spiritual Midwifery, 4th ed., Book Publishing Company, Summertown, Tennessee, USA.

Goffman, E. (1969) The Presentation of Self in Everyday Life, The Penguin Press, Allen Lane, London.

Green, J.M., Curtis, P., Price, H. and Renfrew, M. (1998) Continuing to Care, the Organisation of Midwifery Services in the UK: A Structured Review of the Evidence, Books for Midwives Press, Hale, Cheshire.

Halldorsdottir, S. and Karlsdottir, S.I. (1996) 'Journeying through labour and delivery: perceptions of women who have given birth', Midwifery, Vol. 12, pp.48-61.

Healthcare Commission (2008) Towards Better Births: A Review of Maternity Services in England, Commission for Health Care Audit and Inspection, London.

Hochschild, A.R. (1983) The Managed Heart. Commercialization of Human Feeling, University of California Press, Berkeley, California.

Hochschild, A.R. (2003) The Commercialization of Intimate Life: Notes from Home and Work, University of California Press, Berkeley.

Hunter, B. (2001) 'Emotion work in midwifery: a review of current knowledge', Journal of Advanced Nursing, Vol. 34, No. 4, pp.436-444.

Hunter, B. (2002) Emotion Work in Midwifery: An Ethnographic Study of the Emotional Work Undertaken by a Sample of Student and Qualified Midwives in Wales, Unpublished $\mathrm{PhD}$ Thesis, University of Wales, December, Swansea.

Hunter, B. (2004) 'Conflicting ideologies as a source of emotion work in midwifery', Midwifery, Vol. 20, pp.261-272.

Hunter, B. (2005) 'Emotion work and boundary maintenance in hospital-based midwifery', Midwifery, Vol. 21, pp.253-266.

Hunter, B. (2006) 'The importance of reciprocity in relationships between community-based midwives and mothers', Midwifery, Vol. 22, pp.308-322.

Hunter, B. (2009) 'Mixed messages: midwives' experiences of managing emotion', in Hunter, B. and Deery, R. (Eds.): Emotions in Midwifery and Reproduction, Palgrave Macmillan, Basingstoke, pp.175-191.

Hunter, B. and Deery, R. (Eds.) (2009) Emotions in Midwifery and Reproduction, Palgrave Macmillan, Basingstoke.

Hunter, B. and Smith, P. (2007) 'Emotional labour: just another buzz word? Guest Editorial', International Journal of Nursing Studies, Vol. 44, pp.859-861. 
Hunter, B., Berg, M., Lundgren, I., Olafsdottir, O. and Kirkham, M. (2008) 'Relationships: the hidden threads in the tapestry of maternity care, Guest Commentary', Midwifery, Vol. 24, pp.132-137.

James, N. (1992) 'Care = organisation + physical labour + emotional labour', Sociology of Health and Illness, Vol. 14, No. 4, pp.489-509.

John, V. and Parsons, E. (2006) 'Shadow work in midwifery: unseen and unrecognised emotional labour', British Journal of Midwifery, Vol. 14, No. 5, pp.266-268.

Kennedy, H.P. (1995) 'The essence of nurse-midwifery care: the woman's story', Journal of Nurse-Midwifery, Vol. 40, No. 5, September-October, pp.410-417.

Kirkham, M. (1999) 'The culture of midwifery in the National Health Service in England', Journal of Advanced Nursing, Vol. 30, No. 3, pp.732-739.

Kirkham, M. (2009) 'Emotion work around reproduction: Supportive or constraining?', in Hunter, B. and Deery, R. (Eds.): Emotions in Midwifery and Reproduction, Palgrave Macmillan, Basingstoke, pp.227-239.

Kirkham, M. (Ed.) (2000) The Midwife-Mother Relationship, Macmillan Press, Basingstoke.

Kirkham, M. and Stapleton, H. (2000) 'Midwives' support needs as childbirth changes', Journal of Advanced Nursing, Vol. 32, No. 2, pp.465-472.

Kirkham, M., Morgan, R.K. and Davies, A. (2006) Why Midwives Stay, Dept. of Health, London, available online www.nhsemployers.org www.rcm.org

Kitzinger, J., Green, J. and Coupland, V. (1990) 'Labour relations: midwives and doctors on the labour ward', in Garcia, J., Kilpatrick, R. and Richards, M. (Eds.): The Politics of Maternity Care: Services for Childbearing Women in Twentieth Century Britain, Clarendon Press, Oxford, pp.149-162.

Kitzinger, S. (2008) The New Pregnancy and Childbirth: Choices and Challenges, 4th ed., Dorling Kindersley, London.

Lawler, J. (1991) Behind the Screens: Nursing, Somology and the Problem of the Body, Churchill Livingstone, Edinburgh.

Leap, N. (1997) 'Making sense of 'horizontal violence' in midwifery', British Journal of Midwifery, Vol. 5, p.689.

Leap, N. and Anderson, T. (2004) 'The role of pain in normal birth and the empowerment of women', in Downe, S. (Ed.): Normal Childbirth: Evidence and Debate, Churchill Livingstone, Edinburgh, pp.25-40.

Lipsky, M. (1980) Street-Level Bureaucracy: Dilemmas of the Individual in Public Services, Russell Sage Foundation, New York.

Mackin, P. and Sinclair, M. (1998) 'Labour ward midwives' perceptions of stress', Journal of Advanced Nursing, Vol. 27, pp.986-991.

McCourt, C. and Stevens, T. (2009) 'Relationship and reciprocity in caseload midwifery', in Hunter, B. and Deery, R. (Eds.): Emotions in Midwifery and Reproduction, Palgrave Macmillan, Basingstoke, pp.17-35.

Niven, C. (1994) 'Coping with labour pain: the midwife's role', in Robinson, S. and Thomson, A.M. (Eds.): Midwives, Research and Childbirth, Chapman and Hall, London, Vol. 3, pp.91-119.

Olafsdottir, O.A. (2009) 'Inner knowing and emotion work in the Midwife-woman relationship', in Hunter, B. and Deery, R. (Eds.): Emotions in Midwifery and Reproduction, Palgrave Macmillan, Basingstoke, pp.192-209.

Parsons, T. (1951) The Social System, Free Press, New York.

Redshaw, M., Rowe, R., Hockley, C. and Brocklehurst, P. (2007) Recorded Delivery: A National Survey of Women's Experience of Maternity Care, National Perinatal Epidemiology Unit (NPEU), Oxford.

Rose, G. (2001) Visual Methodologies, Sage, London. 
Sandall, J. (1997) 'Midwives' burnout and continuity of care', British Journal of Midwifery, Vol. 5, No. 2, pp.106-111.

Smith, P. (1992) The Emotional Labour of Nursing, Macmillan, London.

Stevens, T. and McCourt, C. (2002) 'One-to-one midwifery practice part 3: meaning for midwives', British Journal of Midwifery, Vol. 10, No. 2, pp.111-115.

Stewart, M. (2005) 'I'm just going to wash you down': sanitizing the vaginal examination', Journal of Advanced Nursing, Vol. 51, No. 6, pp.587-594.

Twigg, J. (2006) The Body in Health and Social Care, Palgrave Macmillan, London.

Walsh, D. (2006) 'Birth centres, community and social capital', MIDIRS, Vol. 16, No. 1, pp.7-15.

Walsh, D. (2007) Improving Maternity Services. Small is Beautiful - Lessons from a Birth Center, Radcliffe Publishing, Oxford.

Walsh, D., El-Nemer, A. and Downe, S. (2004) 'Risk, safety and the study of physiological birth', in Downe, S. (Ed.): Normal Childbirth: Evidence and Debate, Churchill Livingstone, Edinburgh, pp.103-120.

\section{Notes}

${ }^{1}$ Whilst I acknowledge that some midwives are men and that midwives provide support for fathers as well as mothers, this does not reflect the norm or core of the work.

${ }^{2}$ Bone's study focused on the work of US maternity nurses, rather than midwives. As midwives attend only about $8 \%$ of all US births, maternity nurses are the most common carers for women in labour. For simplicity, I have included maternity nurses in the term 'midwife' for the purpose of this paper; although I acknowledge that there are many important distinctions between these two occupational groups. 